\title{
Solvent-free encapsulation at high pressure in carboxylate-based MOFs
}

Rebeca Monteagudo-Olivan, ${ }^{[a]}$ Lorena Paseta, $^{[a]}$ Grégory Potier, ${ }^{[b]}$ Pilar López-Ram-deViu, ${ }^{[c]}$ Joaquín Coronas* [a]

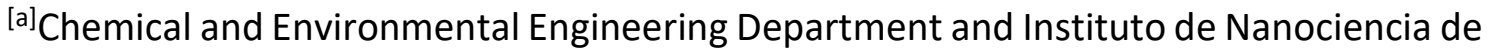
Aragón (INA), Universidad de Zaragoza, 50018 Zaragoza, Spain

${ }^{[b]}$ Département Sciences des Matériaux, Polytech Nantes, 44306 Nantes, France

${ }^{[c]}$ Organic Chemistry Department, Universidad de Zaragoza, and Instituto Universitario de Catálisis Homogénea (Universidad de Zaragoza-CSIC), 50009 Zaragoza, Spain

*Corresponding author: coronas@unizar.es

Rebeca Monteagudo-Olivan

Lorena Paseta

Grégory Potier

Dr. Pilar López-Ram-de-Viu

Prof. Dr. Joaquín Coronas

\section{Abstract}

The solvent-free encapsulation of caffeine and kojic acid is carried out in different carboxylate-based MOFs (MIL-53(Al), UiO-66 and Mg-MOF-74) by high pressure $(0.32$ GPa) contact. This methodology enables fast and ecofriendly encapsulation and gives rise to additive@MOFs with equivalent physical and features to materials obtained by common liquid phase encapsulation processes. It could be applied to other guest-host systems simplifying the procedures, reducing the use and waste of harmful chemicals and approaching the conditions of interest in the industry. The characterization carried out by thermogravimetry, X-ray diffraction, $\mathrm{N}_{2}$ adsorption and ${ }^{13} \mathrm{C} N M R$ provided information about the presence and conformation of the additives in the MOFs. The highest encapsulation values for caffeine (37\%) and kojic acid (32\%) are obtained with MIL-53(Al).

Keywords: Carboxylate ligands, Crystal growth, Green chemistry, Host-guest systems, Metal-organic frameworks 


\section{Introduction}

Metal-organic frameworks (MOFs) are porous crystalline hybrid materials made of metal ions or clusters coordinated with organic linkers to form single or multidimensional structures. ${ }^{1}$ Due to the high surface area of these materials, ${ }^{2}$ their relatively high chemical and thermal stability ${ }^{3,4}$ and the possibility of modifying the pore size and the functionality by changing the metal ion or the organic ligand, ${ }^{5}$ MOFs are useful in a wide range of fields such as catalysis, ${ }^{6}$ adsorption and storage of gases, ${ }^{7}$ selective membranes, ${ }^{8}$ encapsulation, ${ }^{9}$ and medicine. ${ }^{10-13}$

One of the most important families of MOFs corresponds to those based on carboxylate-type ligands. Specifically, MIL-53(Al), UiO-66 and Mg-MOF-74 are very suitable from the encapsulation point of view because of the potential biocompatibility of their corresponding organic linkers and metal centers. ${ }^{14,15} \mathrm{MIL}-53(\mathrm{Al})$ is composed of trivalent metal cations $\mathrm{Al}^{3+}$ interconnected through terephthalate linkers to form a three dimensional framework with rhombus-shape one-dimensional channels. ${ }^{16}$ This MOF has attracted considerable attention due to its high thermal and chemical stability and its "breathing" behavior. This allows it to adapt its porosity, which can vary in the range of $8.5 \times 8.5 \AA$ ( $h t$ form) and $2.6 \times 13.6 \AA$ (/t form), to the size and shape of the guest molecule. ${ }^{16-18}$ This feature makes MIL-53(Al) very interesting in the encapsulation field ${ }^{19}$ and for the delivery of molecules of pharmacological interest. ${ }^{17}$ The second carboxylatebased MOF UiO-66 also shows high stability compared with other MOFs due to the special structure of the corresponding $\mathrm{Zr}^{4+}$-terephthalate, which displays octahedral and tetrahedral cages with triangular pore windows of $6 \AA \AA^{20}$ The third material studied, MgMOF-74, is composed of $\mathrm{Mg}^{2+}$ and 2,5-dihydroxyterephthalate and presents hexagonal channels of $12 \AA .{ }^{21,22}$ This MOF is highly hydrated showing the behavior of a typical high aluminum content zeolite; due to the fact that some water molecules play a structural role, it has low stability in water. ${ }^{23}$ Finally, MIL-101(Cr) is made of terephthalate ligands coordinated to $\mathrm{Cr}^{3+}$ and is well-known as an adsorbent with a high specific surface area; it has cages of $29 \AA$ and $34 \AA$ featuring $12 \AA$ pentagonal and $16 \AA$ hexagonal apertures. ${ }^{24}$ Due to its chromium content, this material is not as biocompatible as the other three materials but has a high adsorption capability. ${ }^{10}$ It is studied here for the purposes of comparison. 
<smiles>Cn1c(=O)c2c(ncn2C)n(C)c1=O</smiles>

Caffeine<smiles></smiles>

Kojic acid

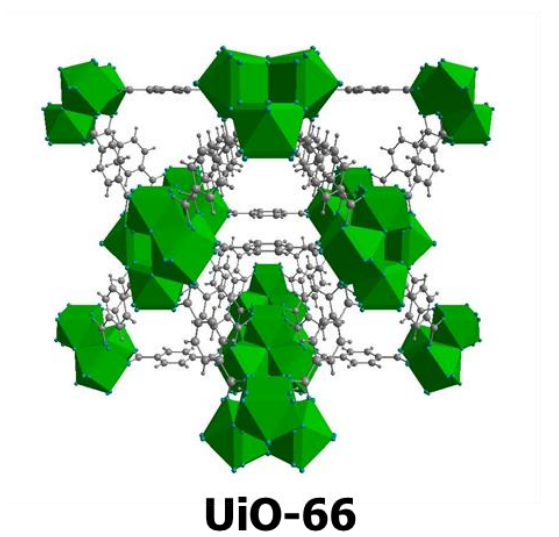

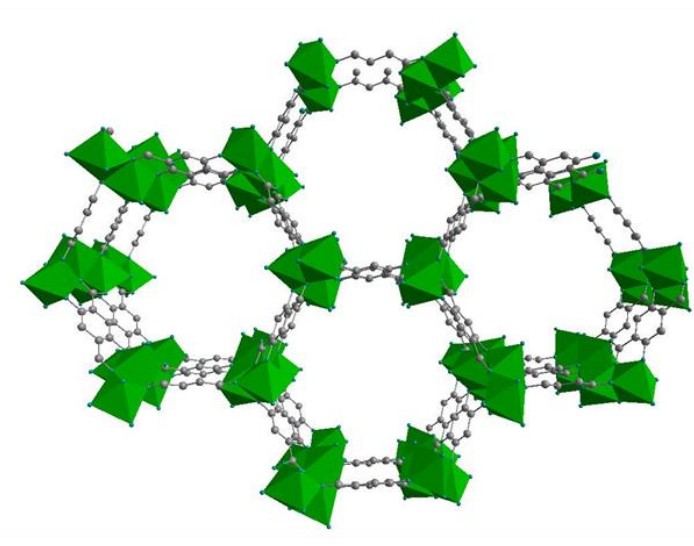

Mg-MOF-74

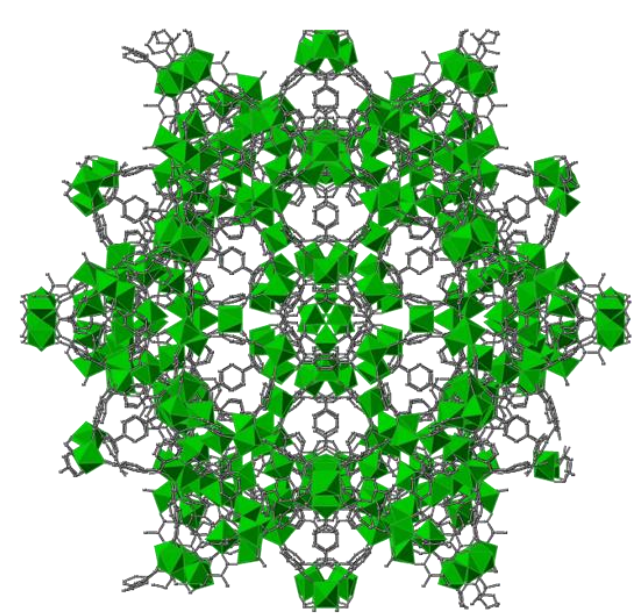

MIL-101(Cr)

Figure 1. Guest and host systems addressed in this work. Atomic color code: carbon (grey), oxygen (blue) and metal coordination (green). These structures were made with Diamond 3.2. and Crystal Maker 9.2.7 using the corresponding CIF files. ${ }^{16,21,25,26}$

Regarding traditional liquid phase encapsulation, there are two different methodologies to encapsulate a drug into a MOF denominated as "multi-step" and

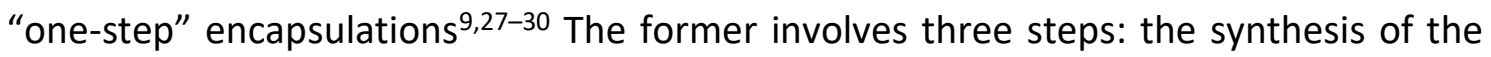
MOF, its subsequent activation, and the encapsulation of the drug by liquid phase adsorption. ${ }^{10,31-34}$ In the latter, the drug is placed together with the reactants in the synthesis media and the MOF grows around the drug. ${ }^{35-39}$ Moreover, after encapsulation, it has been reported the functionalization of MOF particles with different biomolecules ${ }^{40,41}$ and magnetic nanoparticles. ${ }^{42}$ Herein we propose a different and new 
methodology: the solvent-free encapsulation by high pressure $(0.32 \mathrm{GPa})$ contact of additives or drugs (caffeine and kojic acid) with several carboxylate-based MOFs (see Fig. 1). Caffeine can be considered as a model molecule ${ }^{31,35}$ suitable for demonstrating a new encapsulation process. In addition, caffeine is widely known because of its stimulant effect in the central nervous system ${ }^{43}$ but also as a fat reducer in the fields of cosmetics and pharmacology. ${ }^{44}$ To broaden the scope of applicability, a second probe additive, kojic acid, has been used in the present study. This natural compound produced by several fungi is used in low doses for skin lightening ${ }^{45,46}$ in cosmetic products and as an antimelanogenesis agent. ${ }^{47}$ It also has applications as a biocide. ${ }^{48}$ The smaller molecular size of kojic acid (as compared to caffeine) will provide additional insight into the host-guest interaction.

The solvent-free encapsulation process carried out for the first time in this work can be considered as environmentally friendly, in line with others related to the synthesis of MOFs working in continuous mode, ${ }^{49}$ using water as a solvent ${ }^{50}$ or avoiding the use of solvents entirely. ${ }^{49,50}$ We postulate that high pressure favors the diffusion of the additive into the material and therefore the use of any solvent is avoided. The bibliography on the effect of high pressure on MOFs is very scarce. We have previously reported the solvent-less synthesis of ZIF- 8 at high pressure ${ }^{49}$ and the behavior of this MOF at high pressure has also been described with regard to its stability ${ }^{51,52}$ and water intrusion. ${ }^{53}$ Moreover, the contact of MOF Cu-btc with several liquids (alcohols and perfluorotri-N-pentylamine) has been studied up to $8 \mathrm{GPa} .{ }^{54}$ Interestingly, these authors concluded that "the fundamental understanding of high-pressure phenomena in MOFs will play a pivotal role in the advancement of their diverse applied functionalities." Finally, it is worth mentioning that the MOFs studied here, MIL-53(Al), ${ }^{19}$ UiO-66, ${ }^{31,57} \mathrm{Mg}$ MOF-7455 and MIL-101(Cr), ${ }^{56}$ have previously been applied to the conventional encapsulation of different guests. Finally, it has been recently commercialized the MOF based products TruPick and ION-X as systems for food packaging and electronic gas delivery. ${ }^{57}$ In particular, TruPick releases 1-methylcyclepropene (encapsulated in a MOF synthesized by mechanochemistry) that reduces fruit ripening during its storage. This demonstrates that MOF encapsulation is a field with great potential of industrial development.

\section{Results and discussion}

Fig. 2 depicts the typical crystal morphologies of MIL-53(AI), UiO-66, Mg-MOF-74 and MIL-101(Cr) as observed by SEM. These images are consistent with high crystalline materials (as demonstrated below by XRD characterization) which may be considered appropriate for the purpose of studying the solvent free encapsulation of caffeine and kojic acid at high pressure. 


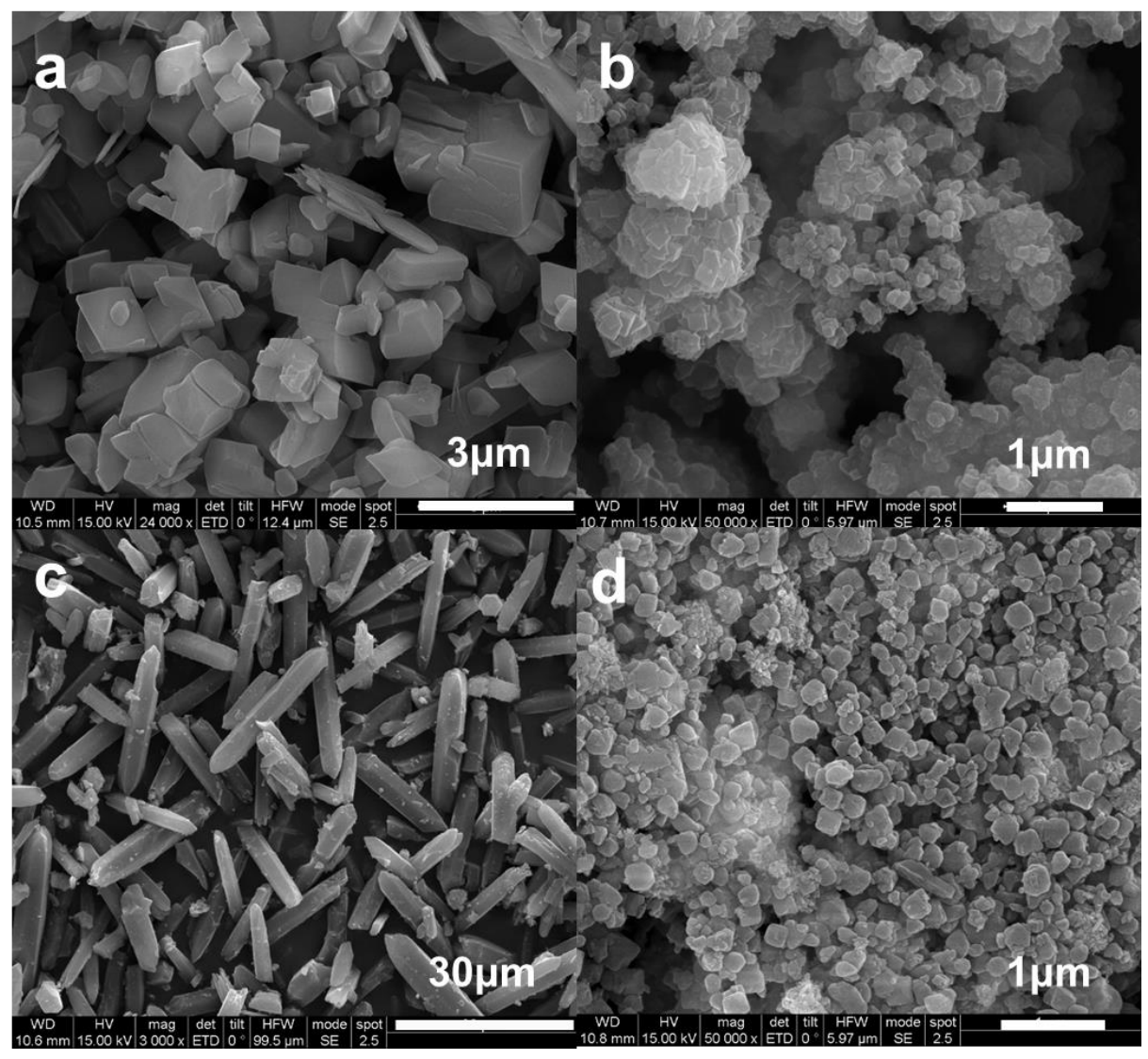

Figure 2. SEM images of the materials MIL-53(Al) (a), UiO-66 (b), Mg-MOF-74 (c) and MIL-101(Cr) (d).

\section{High pressure stability and encapsulation}

In agreement with previous publications dealing with the liquid phase encapsulation of drugs in MOFs, ${ }^{19,31,32,35,37,57,61}$ XRD, $\mathrm{N}_{2}$ adsorption, NMR and TGA techniques were employed to evidence the encapsulation carried out here by the new solvent-free methodology proposed. XRD is qualitative proof of encapsulation, since the guest molecule intensity in its contact with the MOF decreases due the adsorption in the MOF structure. $\mathrm{N}_{2}$ adsorption (to calculate the BET specific surface area) is in line with XRD; if the encapsulation is produced in the MOF porosity, less porosity is available for $\mathrm{N}_{2}$ molecules. NMR demonstrates host-guest interactions and the preservation of the guest chemical nature. Finally, TGA allows a calculation of the encapsulation yield, since encapsulated molecules show a thermal stabilization as compared with external molecules (that have not penetrated in the MOF structure). We did not carry out liquid phase characterizations of our samples to avoid alteration of the solid state encapsulation method.

Fig. 3 shows the diffraction patterns of the different materials before and after $30 \mathrm{~min}$ at $0.32 \mathrm{GPa}$. As an index of the effect of pressure on the decrease of the MOF crystallinity, the FWHM (full width at half maximum height) was calculated for the main 
peaks of the MOFs (MIL-53(Al): $12.5^{\circ}$ - (110) plane, UiO-66: $7.2^{\circ}$ - (111) plane, and MgMOF-74: $11.7^{\circ}$ - (300) plane) before and after pressure exposure. Table S1 shows the obtained results highlighting that the FWHM increased for the pressure treated materials in agreement with a decrease of crystallinity. In particular, MIL-53(AI) and MgMOF-74 seem to be less affected, maintaining their crystalline structures after the high pressure treatment, even though the relative intensities of some peaks varied in the case of MIL-53(AI) probably due to its flexible structure. ${ }^{59}$ Regarding our results with UiO-66, low intensity peaks were still visible in the diffraction pattern after the high pressure treatment but with some noise and evident broadening (Table S1). Finally, strong amorphization was revealed for MIL-101(Cr) after the high pressure treatment treatment, and the FWMH was not calculated. Conspicuously characteristic peaks below $10^{\circ}$ disappeared while most of the others tended to merge into broad peaks. These findings are in agreement with previous works on computational characterization of the mechanical stability of flexible MIL-53(AI) and rigid UiO-66. ${ }^{60,61}$ These works predicted the loss of crystallinity of rigid UiO-66 at $1.83 \mathrm{GPa}$. In turn, this coincides with the experimental work carried out on UiO-66 that concluded that this MOF was not amorphized below $2 \mathrm{GPa}$, even though a gradual loss of crystallinity was detected at moderated pressures $(0.3-1.7 \mathrm{GPa})$ in terms of broadening of the XRD peaks. ${ }^{62}$
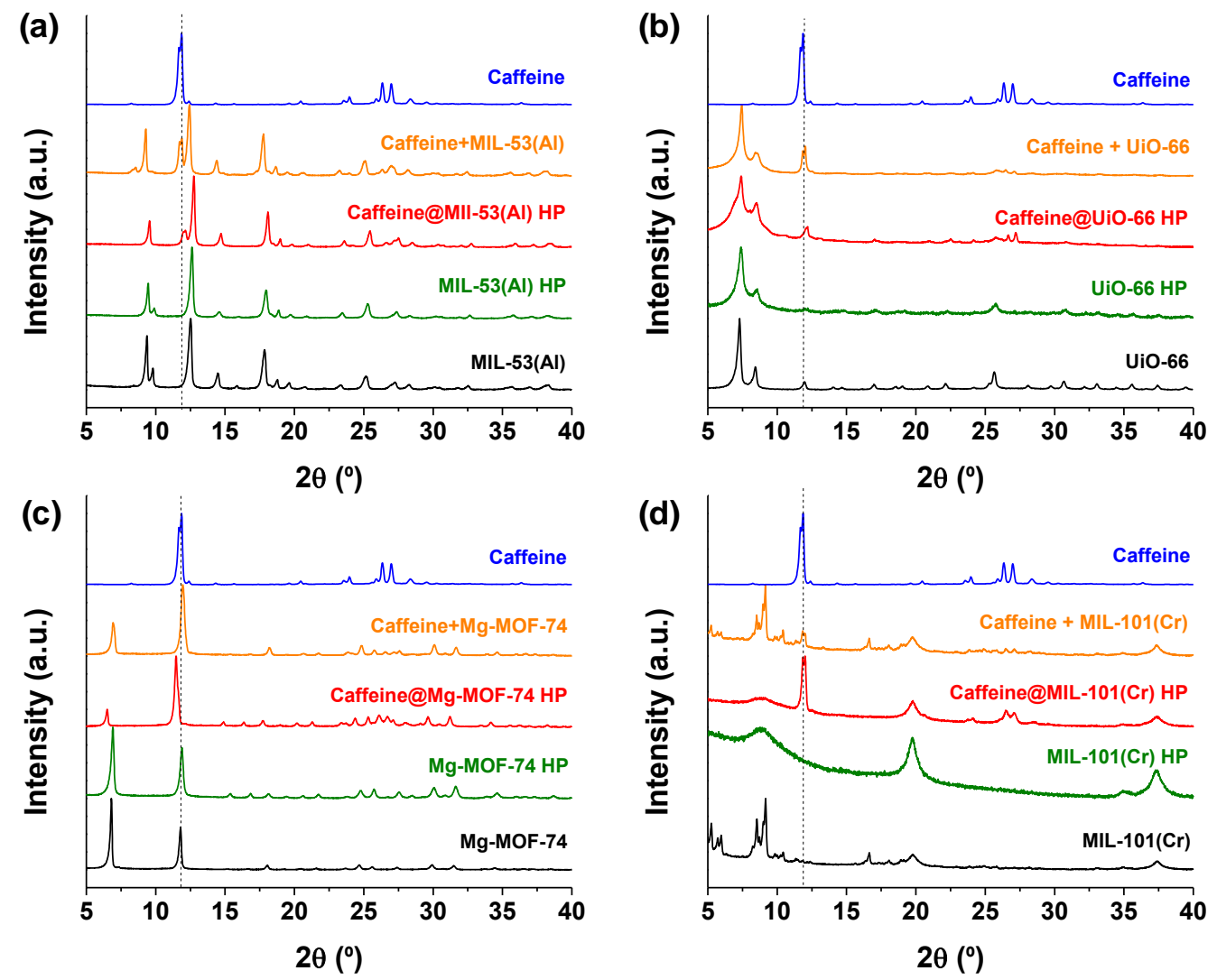

Figure 3. XRD patterns of the different samples (from bottom to top, MOF, MOF after high pressure exposure, caffeine@MOF after high pressure encapsulation, simple mixture of caffeine and MOF, and caffeine) corresponding to: MIL-53(Al) (a), UiO-66 (b), 
Mg-MOF-74 (c), and MIL-101(Cr) (d). Encapsulation at room temperature, $0.32 \mathrm{GPa}$ and 4:1 MOF:caffeine weight ratio.

$\mathrm{XRD}$ is a useful tool for monitoring the effective encapsulation of the additives since the additive intensities would decrease or almost disappear upon guest adsorption in the MOF porosity, i.e. the isolated additives in the MOF porosity would not be able to form detectable crystals. Fig. 3. shows the XRD patterns corresponding to the high pressure encapsulation of caffeine into the different MOFs (MOF:caffeine weight ratio of $4: 1$ ) together with those of the simple blending of caffeine with the MOF (at the same weight ratio) for a proper comparison. Encapsulation of caffeine in MIL-53(Al) and UiO66 produced a clear decrease in the main peak of caffeine at $11.9^{\circ}$, as inferred from the comparison of the XRD patterns corresponding to the simple additive-MOF mixtures and samples caffeine@MIL-53(Al) and caffeine@UiO-66. In consequence, this caffeine peak was considered to follow the encapsulation of caffeine in both MOFs. In this context, Table S2 shows the area ratios corresponding to maximum XRD peaks of additives (caffeine (CAF): $11.9^{\circ}$ and kojic acid (KA): $19.3^{\circ}$ ) and MOFs (MIL-53(Al): $12.5^{\circ}-(110)$ plane, UiO-66: $7.2^{\circ}$ - (111) plane, and Mg-MOF-74: $11.7^{\circ}$ - (300) plane) for simple additive-MOF blending ( $0 \mathrm{GPa}$ ) and encapsulation at $0.32 \mathrm{GPa}$. In all the cases, these area ratios decreased from the simple blending to $0.32 \mathrm{GPa}$ encapsulation. We assume that this ratio decrease is due to the adsorption of caffeine into the MOF pores and therefore the disappearance of external caffeine impregnating the MOFs. Caffeine@MOF products would show the additive pattern if caffeine was present in the form of crystal outside the MOF. The reduction or absence of the characteristic caffeine peaks is consistent with its encapsulation, and the caffeine peaks are more evident when excess caffeine was used (2:1 MOF:caffeine weight ratio instead of 4:1, see Fig. S1).

TGA analysis was used to observe the possible thermal stabilization of additives after encapsulation usually related to their adsorption on the MOF porosity and not to their mere external impregnation. The TGA curve of sample 4:1 in MIL-53(Al) (Fig. S2a) shows only one intermediate step corresponding to thermally stabilized caffeine. In the caffeine@UiO-66 TGA curve (Fig. S2b), two removal steps can be observed due to the external caffeine at ca. $180^{\circ} \mathrm{C}$ and the encapsulated caffeine at ca. $230{ }^{\circ} \mathrm{C}$. The external caffeine step appeared when caffeine was used in high excess, as seen in Fig. S3 for caffeine@MIL-53(Al). The XRD patterns for Mg-MOF-74 are not clear enough to conclude the completion of encapsulation because the most intense peak of caffeine overlaps with one of the peaks of the MOF. In addition, Fig. S2c does not reveal any stabilization for caffeine@MOF-74, in agreement with a mere external impregnation of caffeine on the MOF. In the pressurized sample with MIL-101(Cr), only caffeine peaks are observed, consistent with the great damage that the high pressure produced in this material, suggesting its structural collapse at $0.32 \mathrm{GPa}$. 
Fig. 4 shows similar results for the encapsulation of kojic acid in MIL-53(Al) (Fig. 4a) and UiO-66 (Fig. 4b). Nevertheless, the decrease corresponding to the kojic acid peak area is greater than in the experiments with caffeine as compared to the simple blending, especially in the case of MIL-53(AI) (Table S2). This can be attributed to the more reduced size of kojic acid (MW $142.11 \mathrm{~g} / \mathrm{mol}$ ) as compared to caffeine (MW 194.19 $\mathrm{g} / \mathrm{mol}$ ), considering that it is easier to encapsulate a smaller molecule. However, the chemical interaction estimated in terms of Hansen solubility parameters ${ }^{9,63}$ would favor caffeine-terephthalate interactions (Ra parameter $3.1 \mathrm{MPa}^{0.5}$ ) over those between kojic acid and terephthalate $\left(9.6 \mathrm{MPa}^{0.5}\right)$. TGA curves in Fig. S4 show some thermal stabilization of kojic acid with both MOFs. In the case of Mg-MOF-74, the most intense peak of kojic acid remains in the kojic acid@Mg-MOF-74 (Fig. 4c). The area ratio between the most intense peaks of the additive at $19.3^{\circ}$ and the MOF at $11.7^{\circ}$ is 4.5 for the simple blending and 0.55 for kojic acid@MOF-74 (Table S2), i.e. an important part of the additive disappeared from the external surface during the encapsulation process. Therefore, in this case the change in the relative intensities supports the idea that encapsulation had taken place, in agreement with the corresponding TGA curve in Fig. S4c. MIL-101(Cr) was not used to encapsulate kojic acid due to its lack of high pressure stability, as shown above.
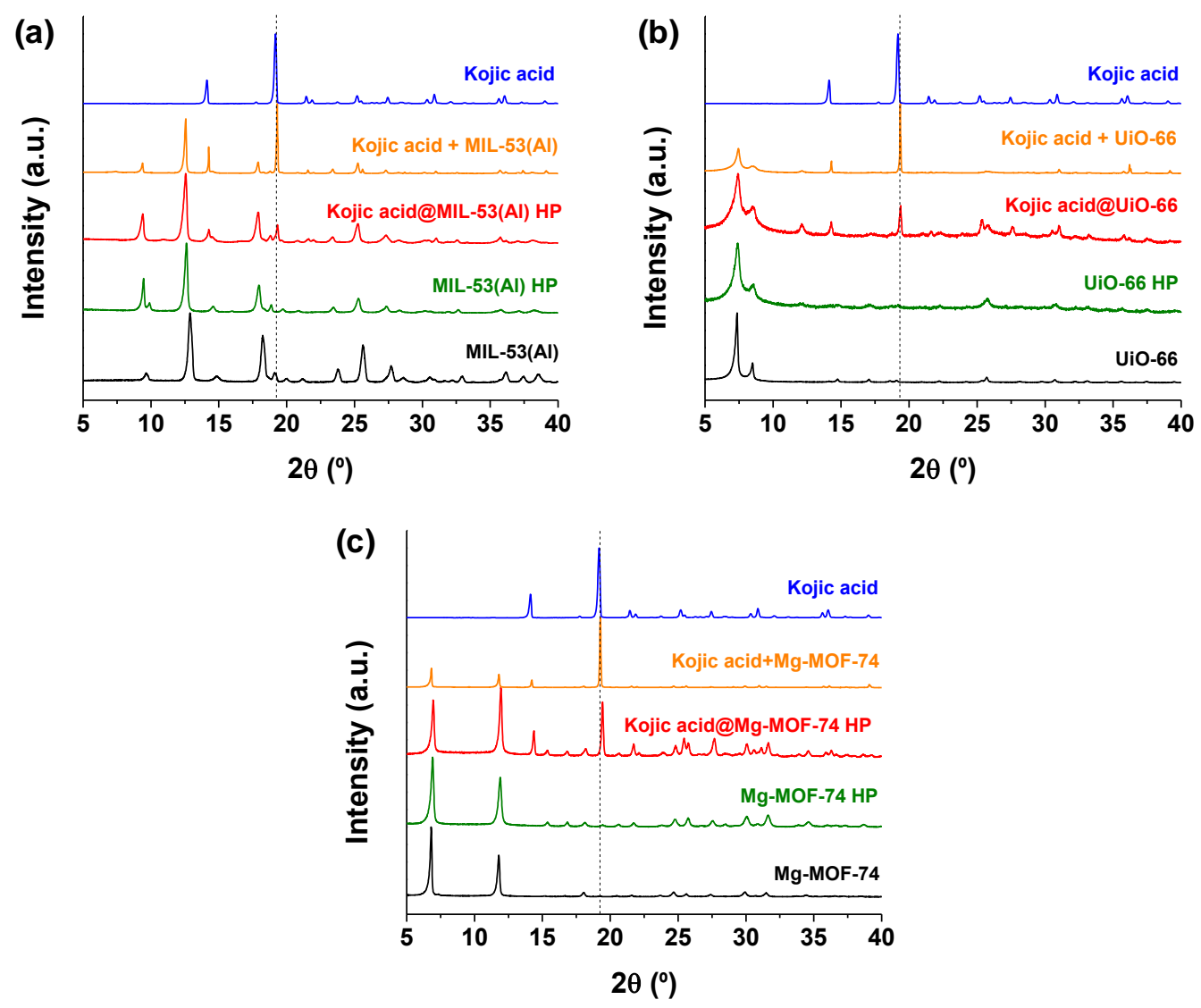

Figure 4. XRD patterns of the different samples (from bottom to top, MOF, MOF after high pressure exposure, kojic acid@MOF after high pressure encapsulation, simple 
mixture of kojic acid and MOF, and kojic acid) corresponding to: MIL-53(Al) (a), UiO-66 (b), and Mg-MOF-74 (c). Encapsulation at room temperature, $0.32 \mathrm{GPa}$ and 4:1 kojic acid:MOF weight ratio

Table 1 shows the BET specific surface area values of some selected samples. First, the effect of high pressure reduced the surface area values for both MIL-53(Al) and UiO-66 from 1140 and $951 \mathrm{~m}^{2} / \mathrm{g}$ to 1016 and $253 \mathrm{~m}^{2} / \mathrm{g}$, respectively. This suggests that the microporosity of MIL-53(Al) was not significantly affected by high pressure, in agreement with the above shown XRD pattern, while that of UiO-66 presents an important reduction, according to the somewhat noisy UiO-66 XRD pattern (suggesting some loss of crystallinity after the high pressure contact). In addition, the decrease of the BET specific surface area due to guest pore filling supports the encapsulation of caffeine and kojic acid in MIL-53(Al) and UiO-66, even though on this last case the effect of guess pore filing may overlap on the structure damage. The decrease of surface area of MIL-53(Al) (1016 m²/g) after the encapsulation of caffeine $\left(336 \mathrm{~m}^{2} / \mathrm{g}\right)$ and kojic acid $\left(9 \mathrm{~m}^{2} / \mathrm{g}\right)$ was consistent with its pores filled with the additives. These values were 52 and $83 \mathrm{~m}^{2} / \mathrm{g}$ for caffeine@UiO-66 and kojic acid@UiO-66, respectively.

Table 1. BET surface area of MIL-53(AI) and UiO-66 after high pressure treatment and high pressure encapsulation of caffeine and kojic acid in both MOFs.

\begin{tabular}{cccc} 
Sample & S $_{\text {BET }}\left(\mathbf{m}^{2} / \mathbf{g}\right)$ & Sample & S $_{\text {BET }}\left(\mathbf{m}^{2} / \mathbf{g}\right)$ \\
\hline MIL-53(Al) & 1140 & UiO-66 & 951 \\
\hline MIL-53(Al) HP & 1016 & UiO-66 HP & 253 \\
\hline Caffeine@MIL-53(Al) & 336 & Caffeine@UiO-66 & 52 \\
\hline Kojic acid@MIL-53(Al) & 9 & Kojic acid@UiO-66 & 83 \\
\hline
\end{tabular}

Solid-state ${ }^{13} \mathrm{C}$ NMR was employed to study the MOF-guest interactions upon encapsulation by high pressure contact. Figures 5 and 6 compare the ${ }^{13} \mathrm{C}$ MAS NMR spectra of caffeine and kojic acid with those of MIL-53(Al) and UiO-66 before and after encapsulation. Some trends were observed analyzing the changes in chemical shifts of additives and terephtalate ligand in additive@MOF materials (Tables S3-S5). In the case of caffeine@MIL-53(Al), the peaks corresponding to the terephthalate MOF linker remained as in the bare MIL-53(Al) (Table S3a). However, the peaks of caffeine were modified once it was encapsulated (see Fig. $5 a$ and Table S4). Interestingly, $C_{2}, C_{4}, C_{5}$, $\mathrm{Me}_{1}$ and $\mathrm{Me}_{6}$ signals shifted upfield, while those for $\mathrm{C}_{7}, \mathrm{C}_{9}$ and $\mathrm{Me}_{3}$ shifted downfield. This can be explained by the anisotropic magnetic behavior of the ligand. There are typical shielding areas due to the aromatic ring effect (affecting to upper and lower parallel planes) and also deshielding zones (anisotropic cone) generated by the carbonyl group. Therefore, the size and shape of the guest molecule and host cages determine a specific and symmetric position of caffeine inside the porosity of MIL-53(Al). An 
arrangement of caffeine-terephthalate planes with $\mathrm{C}_{2}$-axis of terephthalate parallel to an imaginary line bonding $\mathrm{N}_{8}$ and $\mathrm{N}_{1}$ in caffeine ring (Fig. $5 \mathrm{C}$ ) is proposed for caffeine@MIL-53(Al). However, the symmetry of the MOF linker is not affected and the ${ }^{13} \mathrm{C}$ NMR peaks are similar to those of the bare MIL-53(Al).

Caffeine@UiO-66 exhibits ${ }^{13} \mathrm{C}$ NMR peaks corresponding to the MOF ligand wider as compared to the bare UiO-66 (Figure 5b). This agrees with the loss of crystallinity after the encapsulation process and with the XRD results and BET specific surface area values above discussed. Nevertheless, taking into account the observed changes in the chemical shifts of caffeine and ligand signals, a complementary explanation is proposed. A similar trend of caffeine signals in both MIL-53 and UiO-66 (Table S4) shows a parallel arrangement between additive and terephthalate ligand in both MOFs. Simultaneously, the slight tendency to downfield of broadened carboxylate signal of ligand (a shoulder can even be observed at $172.3 \mathrm{ppm}$, Table S3b) next to a deshielding of $\mathrm{C}_{\text {orto }}$ suggests the formation of hydrogen bonds that involve the carboxylic group of terephthalate. Simultaneously, the amorphization of caffeine@UiO-66 and the different kind of hostguest interactions may increase the anisotropy of the host giving rise to the observed broad peaks.

(a)

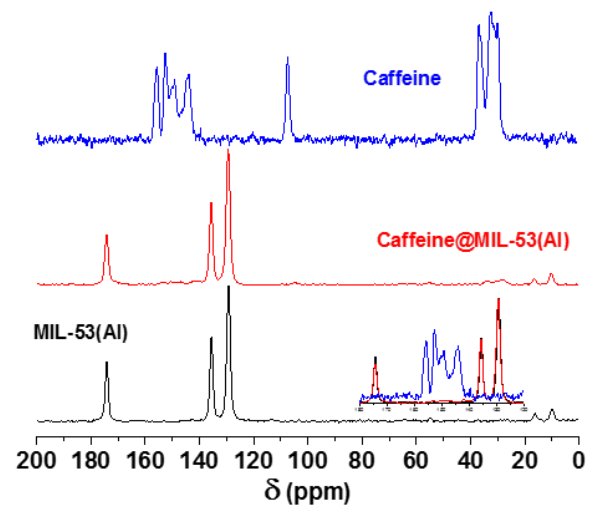

(b)

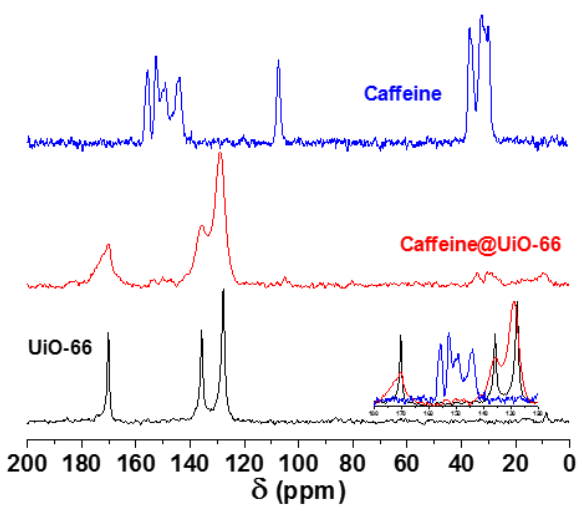

(c)

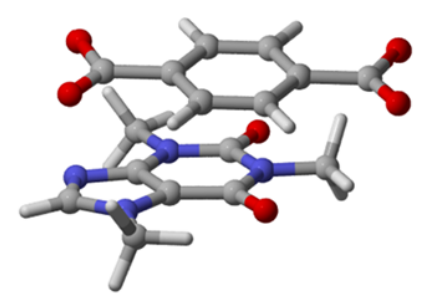

Figure $5 .{ }^{13} \mathrm{C}$ MAS NMR spectra of the different samples (from bottom to top, MOF, caffeine@MOF after high pressure encapsulation and caffeine) corresponding to: MIL53(Al) (a), and UiO-66 (b). Encapsulation at room temperature, 0.32 GPa and 4:1 caffeine:MOF weight ratio. Proposed arrangement of caffeine-terephthalate planes with $\mathrm{C}_{2}$-axis of terephthalate parallel to an imaginary line bonding $\mathrm{N}_{8}$ and $\mathrm{N}_{1}$ in caffeine ring. Color code: upfield atoms (blue) and downfield atoms (red) (c). 
For kojic acid@MIL-53(Al) the terephthalate ${ }^{13} \mathrm{C}$ NMR signals were also widened (see Fig. 6a and Table S5) as compared to those of kojic acid and MIL53(Al). This is attributable to the host-guest interactions because the MIL-53(Al) structure was preserved after the high pressure treatment, in agreement with both XRD and $\mathrm{N}_{2}$ adsorption characterizations. The chemical ${ }^{13} \mathrm{C}$ NMR signals of terephthalate in kojic acid@MIL-53(Al) followed a different pattern from those of caffeine@MIL-53(Al) and kojic acid@UiO-66 (Table S5), and carboxylate and $\mathrm{C}_{\text {orto }}$ signals of ligand shifted upfield. This behavior suggests that the presence of the additive modifies the metalterephthalate interactions. It has been described that changes in binding modes of the acetate group in metallic clusters can be determined using the solid-state ${ }^{13} \mathrm{C}$ NMR: carboxylate peak shifts upfield when it changes from chelating mode to bidentate or monodentate bridge. ${ }^{64}$ This change in metal-terephthalate binding must be due to the effect of kojic acid. It is known that kojic acid forms stable chelates with metal acetate salts. ${ }^{65}$ Nevertheless, the suggested interactions are reversible, as the thermodiffractometry experiments suggests (see below).

Finally, the chemical ${ }^{13} \mathrm{C}$ NMR peaks of terephthalate in kojic acid@UiO-66 did not broaden suggesting ordered host-guest interactions (Fig. $6 \mathrm{~b}$ and Table S5). Carboxylate and $C_{\text {orto }}$ signals of ligand were deshielded evidencing the formation of hydrogen bonds between carboxylic groups of terephthalate and hydroxyl groups of kojic acid. A slightly shielding of carbon atoms of kojic acid suggests an additional stacking of the aromatic rings of both structures in parallel planes. The behavior of carbonyl $\mathrm{C}_{4}$ in kojic acid is different because it is the most electronically affected by the intermolecular hydrogen bond with terephthalate, since it must loss its intramolecular hydrogen bond. These kojic acid-UiO-66 interactions could preserve the symmetry of the linker and the ordered structure of MOF, in agreement with ${ }^{13} \mathrm{C}$ NMR spectra.

(a)

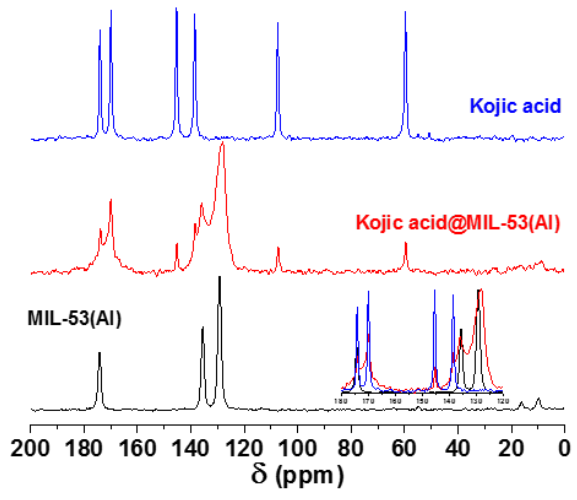

(b)

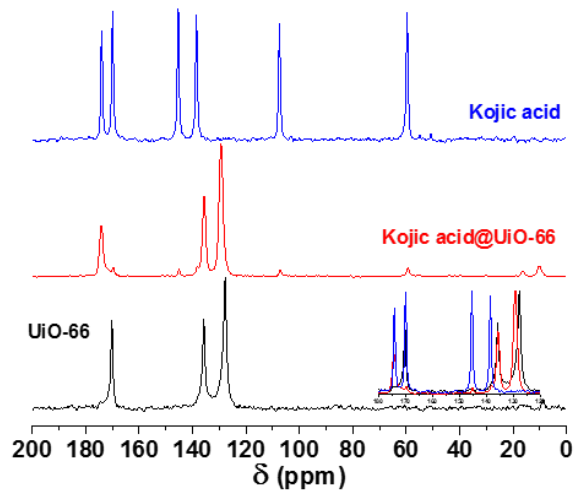

Figure 6. ${ }^{13} \mathrm{C}$ MAS NMR spectra of the different samples (from bottm to top, MOF, kojic acid@MOF after high pressure encapsulation and kojic acid) corresponding to MIL53(Al)(a), and UiO-66 (b). Encapsulation at room temperature, 0.32 GPa and 4:1 kojic acid:MOF weight ratio. 
Table 2 shows the encapsulation values achieved with the different MOFs. The calculation was made per g dry MOF, i.e. excluding from the curves in Figs. S1 and S3 solvent (in principle water from moisture) and external (non-encapsulated) additive weight losses below ca. $100^{\circ} \mathrm{C}$ and above the removal temperature of the pure additive, respectively. The material that produced the best results was MIL-53(Al), with $37 \%$ and $32 \%$ loadings for caffeine and kojic acid, respectively. This can be related to its exceptional stability against mechanical treatment and the flexibility of its structure, ${ }^{16}$ which facilitates the successful encapsulation of the two different additives, caffeine and kojic acid. Additionally, UiO-66 showed $15 \%$ and $22 \%$ loadings for caffeine and kojic acid, respectively, but the loadings (probably due to its small porosity and lack of flexibility) and the mechanical stability (see the above XRD discussion) were lower than in the case of MIL-53(Al). Mg-MOF-74 has been demonstrated to be stable at high pressure and the encapsulation seems to be achieved only for kojic acid (24\%) under the experimental conditions tested. The encapsulation in Mg-MOF-74 involves the exchange of water (present in the as-made MOF, as shown in Fig. S2c with the first step around $100{ }^{\circ} \mathrm{C}$ ) by the desired guest, and only kojic acid was able to produce such an exchange. Table 2 also shows the number of molecules of additive per unit cell (u.c.): 1.6 and 1.3 molecules/u.c. for caffeine@MIL-53(Al) and caffeine@UiO-66, respectively, and 1.9, 2.5 and 2.1 molecules/u.c. for kojic acic@MIL-53(Al), kojic acid@UiO-66 and kojic acid@MgMOF-74, respectively. For the corresponding calculations, the unit cell formulae of $\mathrm{C}_{32} \mathrm{Al}_{4} \mathrm{O}_{20} \mathrm{H}_{20}$ (MIL-53(AI)), ${ }^{16} \mathrm{C}_{192} \mathrm{Zr}_{24} \mathrm{O}_{120} \mathrm{H}_{96}(\mathrm{UiO}-66)^{20}$ and $\mathrm{C}_{16} \mathrm{Mg}_{16} \mathrm{O}_{40} \mathrm{H}_{8}$ (Mg-MOF-74) ${ }^{25}$ were used .This means that the wt\% loading corresponding to one molecule per u.c. is 23,12 and 16 wt\% in case of caffeine and $17,8.7$ and 12 wt\% in case of kojic acid for for MIL-53(Al), UiO-66 and Mg-MOF-74, respectively.

Table 2. Caffeine and kojic acid encapsulation in different MOFs at $0.32 \mathrm{GPa}$ with 4:1 MOF:guest weight ratio and room temperature in (g guest/g dry MOF)·100 and number of additive molecules per unit cell (molec./u.c.). The unit cell formulae of $\mathrm{C}_{32} \mathrm{Al}_{4} \mathrm{O}_{20} \mathrm{H}_{20}$, $\mathrm{C}_{192} \mathrm{Zr}_{24} \mathrm{O}_{120} \mathrm{H}_{96}$ and $\mathrm{C}_{16} \mathrm{Mg}_{16} \mathrm{O}_{40} \mathrm{H}_{8}$ have been considered for MIL-53(Al), ${ }^{16}$ UiO-66 ${ }^{20}$ and Mg-MOF-74, ${ }^{25}$ respectively, for the calculations.
Guest
MIL-53(AI)
Uio-66
Mg-MOF-74

\begin{tabular}{ccccccc}
\hline & & 1.6 & & 1.3 & 0 \\
Caffeine & $37 \%$ & molec./u.c. & $15 \%$ & molec./u.c. & $0 \%$ & molec./u.c. \\
\hline \multirow{2}{*}{ Kojic acid } & $32 \%$ & molec./u.c. & $22 \%$ & molec./u.c. & $24 \%$ & molec./u.c. \\
\hline
\end{tabular}

\subsection{Effect of temperature and pressure on the encapsulation in MIL-53(Al)}

MIL-53(Al) is sensitive to temperature ${ }^{16,66}$ and a sufficiently high temperature may help the desorption of the guest. We have therefore observed the effect of 
different temperatures on high pressure encapsulation samples in thermodiffractometry experiments. Fig.7 shows the diffraction patterns measured in the $25-300{ }^{\circ} \mathrm{C}$ range. When increasing the temperature from 50 to $100{ }^{\circ} \mathrm{C}$, the caffeine@MIL-53 sample changed its structure from the hydrated, low temperature form (MIL-53(Al) It) to the high temperature form (MIL-53(Al) $h t,{ }^{16}$ maintained upon heating up to $300^{\circ} \mathrm{C}$ ), while caffeine peaks are scarcely apparent. However, the TGA analyses carried out on pure caffeine and caffeine@MIL-53 are consistent with the removal of caffeine at a temperature higher than $100{ }^{\circ} \mathrm{C}$ (Fig. S2a). This suggests that the action of the high temperature favors the encapsulation of caffeine guest molecules placed in the surroundings of caffeine@MIL-53. This can be considered as a process in series. First, the high temperature increases the mobility of caffeine molecules already encapsulated, but occupying mostly external crystal pores and eventually reaching empty internal crystal pores. Second, caffeine molecules impregnating the external surfaces of MIL-53 crystals penetrate inside the porous structure.

In the case of the kojic acid@MIL-53 sample, the phase transition is also observed (Fig. 7). Additionally, the complexity of the peaks is considerably increased at $150^{\circ} \mathrm{C}$. We assume that the molecule of kojic acid interacts with the functional groups in the pores and, as occurs with water molecules, the structure changes according to this stimulus. The recovery of the structure of $\mathrm{MIL}-53$ (Al) ht upon heating at $300{ }^{\circ} \mathrm{C}$ is consistent with a reversible encapsulation process in both caffeine@MIL-53 and kojic acid@MIL-53 samples. These results are completed with the thermodiffractometries corresponding to the encapsulations of caffeine and kojic acid on UiO-66 and Mg-MOF-74 (Fig. S5).
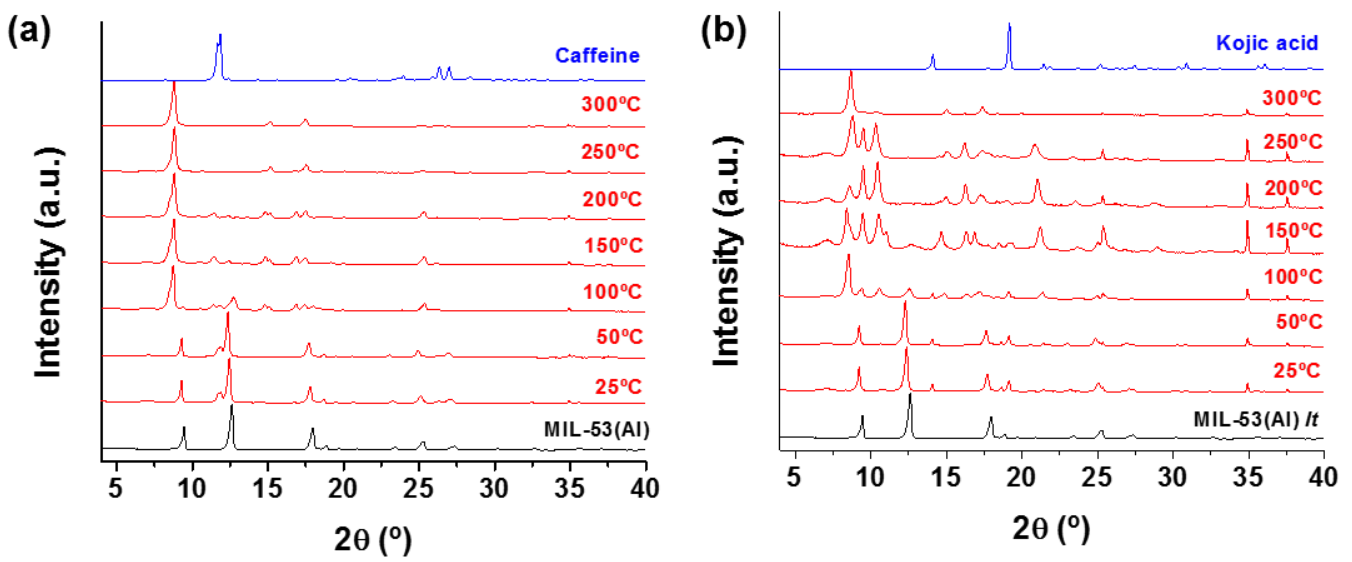

Figure 7. Thermodiffractometry in air with a heating ramp of $10^{\circ} \mathrm{C} / \mathrm{min}$ of caffeine@MIL53(Al) (a) and kojic acid@MIL-53 (b). Encapsulation at 0.32 GPa and with a 4:1 MOF:guest weight ratio.

Once demonstrated the new solventless application, to gain insight into the encapsulation at high pressure and the impact of temperature, the influence of a small amount of ethanol in the encapsulation of caffeine in MIL-53(Al) was investigated. After 
the addition, the mixture was still a solid considering the small amount of ethanol used in the experiment. This would improve the additive-MOF contact and hence the diffusion and dispersion of the additive into the pores of the MOF. Fig. S6 shows a more complex XRD pattern compared to those of the as-made material and those encapsulated without ethanol (Fig. 3a). As mentioned above, MIL-53(Al) "breathes" depending on the presence of guest molecules in the pores of the MOF, and as a consequence the diffraction pattern was modified. In this experiment, caffeine and ethanol were simultaneously adsorbed on the material evidencing changes in the XRD intensities, consistent with an opening (as compared with the It as-made material) of the porosity as observed for MIL-53(Cr) when adsorbing methanol and ethanol. ${ }^{67}$

The sample caffeine@MIL-53(Al), obtained in the presence of ethanol, was used in a thermodiffractometry experiment carried out under vacuum. Together with this sample, Fig. 56 shows for the purposes of comparison the XRD patterns of caffeine and MIL-53(Al) at the initial conditions previous to the encapsulation, i.e. in the form known as $/ t .{ }^{16}$ Upon heating at $200{ }^{\circ} \mathrm{C}$, the ethanol was desorbed from the MOF and the XRD adapted the $h t$ form for MIL-53(Al). ${ }^{68}$ After subsequent heating at $300{ }^{\circ} \mathrm{C}$, caffeine should have been removed (in agreement with the TGA shown in Fig. S7); however, no further changes were observed in the XRD pattern.

Finally, even though most of the encapsulation experiments were carried out at $0.32 \mathrm{GPa}$, Tables S6 and S7 and Fig. S8 show the encapsulation of caffeine and kojic acid on MIL-53(Al) at three different pressures from 0.32 to $0.64 \mathrm{GPa}$. The decrease of the percentage of encapsulation (MIL-53(AI)) and the similar values of BET specific surface area observed with increasing pressure suggest that $0.32 \mathrm{GPa}$ was the optimum working pressure. This pressure relates to a good balance between the loss of MOF crystallinity and the encapsulation efficiency.

\section{Conclusions}

We have demonstrated the simple and ecofriendly encapsulation of caffeine in two different MOFs by means of a high pressure application (0.32 GPa). This simple procedure may be of considerable industrial interest considering its speed and the fact that it does not need further purification (i.e. separation from the encapsulation dispersion as in the case of conventional liquid phase encapsulation). This minimizes the use of solvents and the potential generation of waste. Moreover, the solvent free, high pressure approach has been carried out with three MOFs (MIL-53(Al), UiO-66 and MgMOF-74, since MIL-101(Cr) was strongly amorphized upon high pressure exposure) and two guests (caffeine and kojic acid), the best results being obtained with MIL-53(Al) due probably to its high structural flexibility that helps the diffusion of caffeine under the pressure effect. The XRD characterization demonstrated the encapsulation qualitatively, while TGA allowed to estimate of the amount of drug encapsulated in every case. In addition, the decrease of the BET specific surface area of MOFs after the encapsulation 
of caffeine and kojic acid was consistent with its pores filled with the additives. The ${ }^{13} \mathrm{C}$ MAS NMR spectra of the additive@MOF materials support the preservation of the chemical nature of caffeine and kojic acid upon high pressure encapsulation, suggesting in some cases (caffeine@MIL-53(AI) and kojic acid@UiO-66) relatively ordered hostguest interactions. The combination of the four characterization techniques demonstrated both the qualitative and quantitative caffeine and kojic acid encapsulation in the studied MOFs using the at high pressure conditions in solvent-free conditions. Finally, the results achieved with this research, obtained through a green process, allow one to say that MOF encapsulation is a field with great potential of industrial development where the costs and environmental impacts can be minimized.

\section{Experimental section}

\section{Synthesis of materials}

Synthesis of MIL-53(Al). In a typical synthesis, ${ }^{16} 5.20 \mathrm{~g}$ of aluminum nitrate nonahydrate (13.9 mmol, $\mathrm{Al}\left(\mathrm{NO}_{3}\right)_{3} \cdot 9 \mathrm{H}_{2} \mathrm{O}$, Sigma Aldrich, $\left.\geq 98 \%\right)$ together with $1.12 \mathrm{~g}$ of terephthalic acid (6.7 mmol, $\mathrm{H}_{2} \mathrm{BDC}$, Sigma Aldrich, 98\%) were dispersed in $100 \mathrm{~mL}$ of distilled water and placed in a Teflon-lined stainless steel autoclave for 3 days at $220^{\circ} \mathrm{C}$. The resulting product was recovered by centrifugation at $10,000 \mathrm{rpm}$ for $10 \mathrm{~min}$, washed once with ethanol followed by centrifugation under the same conditions and dried overnight at $65^{\circ} \mathrm{C}$. The solid was activated by calcination at $380^{\circ} \mathrm{C}$ for $24 \mathrm{~h}$.

Synthesis of UiO-66. Following a previous report, ${ }^{20} 0.508 \mathrm{~g}$ of $\mathrm{ZrCl}_{4}(2 \mathrm{mmol}$, Sigma Aldrich, $\geq 99,5 \%$ ) and $0.667 \mathrm{~g}$ of terephthalic acid ( $4 \mathrm{mmol}, \mathrm{H}_{2} \mathrm{BDC}$, Sigma Aldrich, $98 \%$ ) were mixed with $100 \mathrm{~mL}$ of dimethylformamide (DMF) and the resulting solution placed in a Teflon-lined stainless steel autoclave for $24 \mathrm{~h}$ at $120^{\circ} \mathrm{C}$. The white product was recovered by centrifugation at $10,000 \mathrm{rpm}$ for $10 \mathrm{~min}$ and washed once with ethanol followed by centrifugation under the same conditions. The solid was activated by calcination at $300^{\circ} \mathrm{C}$ for $4 \mathrm{~h}$.

Synthesis of Mg-MOF-74. As reported elsewhere, ${ }^{21} 0.149 \mathrm{~g}$ of 2,5dihydroxyterephthalic acid $(0.75 \mathrm{mmol}$, DOBDC, TCl, >98\%) was dissolved in $10 \mathrm{~mL}$ of THF after which $3 \mathrm{~mL}$ of a $1 \mathrm{M} \mathrm{NaOH}$ aqueous solution was added. A solution of $0.384 \mathrm{~g}$ of magnesium nitrate hexahydrate ( $1.5 \mathrm{mmol}, \mathrm{Mg}\left(\mathrm{NO}_{3}\right)_{2} \cdot 6 \mathrm{H}_{2} \mathrm{O}$, Sigma Aldrich, $\left.98 \%\right)$ in 3 $\mathrm{mL}$ of distilled water was added and the resulting mixture placed in a Teflon-lined stainless steel autoclave for 3 days at $110{ }^{\circ} \mathrm{C}$. The yellow product was recovered by centrifugation at $10,000 \mathrm{rpm}$ for $10 \mathrm{~min}$ and washed several times with methanol followed by centrifugation under the same conditions. The product was dried overnight at room temperature.

Synthesis of MIL-101(Cr). Following a previous report, ${ }^{69} 0.83 \mathrm{~g}$ of terephthalic acid ( $5 \mathrm{mmol}, \mathrm{H}_{2} \mathrm{BDC}$, Sigma Aldrich, $98 \%$ ) and $2.00 \mathrm{~g}$ of chromium nitrate nonahydrate (5 mmol, $\mathrm{Cr}\left(\mathrm{NO}_{3}\right)_{3} \cdot 9 \mathrm{H}_{2} \mathrm{O}$, Sigma Aldrich, 98\%) were mixed in $25 \mathrm{~mL}$ of distilled water and placed in a Teflon-lined stainless steel autoclave for $8 \mathrm{~h}$ at $220{ }^{\circ} \mathrm{C}$. The green product 
was recovered by centrifugation at $10,000 \mathrm{rpm}$ for $10 \mathrm{~min}$ and washed with methanol followed by centrifugation under the same conditions. The solid was activated by treatment in DMF for $24 \mathrm{~h}$ at $150{ }^{\circ} \mathrm{C}$ and then boiled in a reflux in methanol overnight. The product was dried at room temperature for $8 \mathrm{~h}$.

\section{$\underline{\text { High pressure encapsulation }}$}

The procedure for the high pressure encapsulation was as follows: $100 \mathrm{mg}$ of the MOF material and $25 \mathrm{mg}$ of additive (weight ratio 4:1) were mixed together by $1 \mathrm{~min}$ hand shaking in a vial. The mixture was then placed at room temperature inside the metal cylinder of a hydraulic press (Specac 25.011). After insertion of the metal piston, pills were compacted under a pressure of 0.32-0.64 GPa for $30 \mathrm{~min}$. The compacted material pill was gently milled into powder and is referred to here as additive@MOF. Blanks corresponding to the MOFs and additives separately exposed to high pressure were produced under the same conditions. Additionally, in the case of MIL-53(AI), 2:1 and 1:1 MOF:additive weight ratios were contacted at high pressure. Finally, a 2:1 weight ratio experiment was carried out at $0.32 \mathrm{GPa}$ for $30 \mathrm{~min}$ with the addition of 20 $\mathrm{mg}$ (ca. $0.025 \mathrm{~mL}$ ) of ethanol, i.e. using a 2:1:0.4 MOF:additive:ethanol weight ratio.

\section{Characterization}

Scanning electron microscopy (SEM, FEI Inspect F50) observation of the powder samples was carried out with a voltage 2-15 kV and after Pt coating. Powder X-ray diffraction (XRD) was carried out at room temperature in a Siemens diffractometer with a copper anode and a graphite monochromator so as to select $\mathrm{C} U-K \alpha_{1}$ radiation $(\lambda=1.540$ $\AA$ ). Data were collected in the $4-40^{\circ} 2 \theta$ range, and the scanning rate was $0.03^{\circ} / \mathrm{s}$. This technique was used to check the crystallinity after the process and to observe the decrease of additive peaks in high pressure contact encapsulation due to the reduction of the external additive. Nitrogen adsorption isotherm and BET specific surface area were measured with a Micrometrics TriStar 3000 with a previous degasification at 150 ${ }^{\circ} \mathrm{C}$ for $5 \mathrm{~h}$. The ${ }^{13} \mathrm{C}$ NMR spectra were achieved with cross-polarized magic angle spinning solid nuclear magnetic resonance (CP MAS-NMR) in a Bruker Avance III WB 400. Thermogravimetric analyses (TGA) were performed using Mettler Toledo TGA/SDTA $851 \mathrm{e}$ instrument. The samples were put in $70 \mu \mathrm{L}$ alumina pans and heated up to $700{ }^{\circ} \mathrm{C}$ with a heating rate of $10^{\circ} \mathrm{C} / \mathrm{min}$ in air. Thermodiffractometry was performed under air in a furnace coupled to a Siemens diffractometer with a copper anode and a graphite monochromator to select the same $\mathrm{Cu}-\mathrm{K}_{\alpha 1}$ radiation. Each XRD pattern was recorded for $20 \mathrm{~min}$ in the $3-40^{\circ} 2 \theta$ range with a $0.01^{\circ} / \mathrm{s}$ scanning rate at $25,50,100,150,200,250$ and $300^{\circ} \mathrm{C}$ with a heating rate of $10^{\circ} \mathrm{C} / \mathrm{min}$. 


\section{Acknowledgements}

The authors acknowledge financial support from the Aragón Government (DGA, T43$17 R)$ and the European Social Fund. The microscopy works were carried out in the Laboratorio de Microscopías Avanzadas at the Instituto de Nanociencia de Aragón (LMAINA, Universidad de Zaragoza). The authors would like to acknowledge the use of the Servicio General de Apoyo a la Investigación-SAI (Universidad de Zaragoza). Prof. Dr. Steven Abbott is thanked for providing Hansen solubility parameters.

\section{References}

1 G. Férey, Chem. Soc. Rev., 2008, 37, 191-214.

2 J. L. C. Rowsell and O. M. Yaghi, Angew. Chem., Int. Ed., 2005, 44, 4670-4679 ; Angew. Chem., 2005, 117, 48

3 N. Li, J. Xu, R. Feng, T.-L. L. Hu and X.-H. H. Bu, Chem. Commun., 2016, 52, 85018513.

4 J. B. DeCoste, G. W. Peterson, H. Jasuja, T. G. Glover, Y. Huang and K. S. Walton, J. Mater. Chem. A, 2013, 1, 5642-5650.

5 H. Li, M. Eddaoudi, M. O'Keeffe and O. M. Yaghi, Nature, 1999, 402, 276-279.

6 J. Lee, O. K. Farha, J. Roberts, K. a Scheidt, S. T. Nguyen and J. T. Hupp, Chem. Soc. Rev., 2009, 38, 1450-1459.

7 J.-R. Li, R. J. Kuppler and H.-C. Zhou, Chem. Soc. Rev., 2009, 38, 1477-1504.

8 S. Sorribas, P. Gorgojo, C. Téllez, J. Coronas and A. G. Livingston, J. Am. Chem. Soc., 2013, 135, 15201-15208.

9 L. Paseta, G. Potier, S. Abbott and J. Coronas, Org. Biomol. Chem., 2015, 13, 1724-1731.

10 P. Horcajada, T. Chalati, C. Serre, B. Gillet, C. Sebrie, T. Baati, J. F. Eubank, D. Heurtaux, P. Clayette, C. Kreuz, J.-S. Chang, Y. K. Hwang, V. Marsaud, P.-N.

Bories, L. Cynober, S. Gil, G. Férey, P. Couvreur and R. Gref, Nat. Mater., 2010, 9, 172-178.

11 S. Wuttke, M. Lismont, A. Escudero, B. Rungtaweevoranit and W. J. Parak, Biomaterials, 2017, 123, 172-183.

12 M. Lismont, L. Dreesen and S. Wuttke, Adv. Funct. Mater., 2017, 27.

13 P. Horcajada, R. Gref, T. Baati, P. K. Allan, G. Maurin, P. Couvreur, G. Férey, R. E. Morris and C. Serre, Chem. Rev., 2012, 112, 1232-1268.

14 V. André and S. Quaresma, eds. F. Zafar and E. B. T.-M.-O. F. Sharmin, InTech, Rijeka, 2016, p. Ch. 07.

15 A. C. McKinlay, R. E. Morris, P. Horcajada, G. Férey, R. Gref, P. Couvreur and C. Serre, Angew. Chem., Int. Ed., 2010, 49, 6260-6266; Angew.Chem., 2010, 122, 6400-6406.

16 T. Loiseau, C. Serre, C. Huguenard, G. Fink, F. Taulelle, M. Henry, T. Bataille and G. Férey, Chemistry, 2004, 10, 1373-1382.

17 P. Horcajada, C. Serre, G. Maurin, N. A. Ramsahye, F. Balas, M. Vallet-Regí, M. Sebban, F. Taulelle and G. Férey, J. Am. Chem. Soc., 2008, 130, 6774-6780.

18 C. Serre, C. Mellot-Draznieks, S. Surblé, N. Audebrand, Y. Filinchuk and G. Férey, 
Science (80-. )., 2007, 315, 1828-1831.

L. Paseta, E. Simón-Gaudó, F. Gracia-Gorría and J. Coronas, Chem. Eng. J., 2016, 292, 28-34.

20 J. H. Cavka, S. Jakobsen, U. Olsbye, N. Guillou, C. Lamberti, S. Bordiga and K. P. Lillerud, J. Am. Chem. Soc., 2008, 130, 13850-13851.

21 P. D. C. Dietzel, R. Blom and H. Fjellvåg, Eur. J. Inorg. Chem., 2008, 3624-3632.

22 S. R. Caskey, A. G. Wong-Foy and A. J. Matzger, J. Am. Chem. Soc., 2008, 130, 10870-10871.

23 Y. Jiao, C. R. Morelock, N. C. Burtch, W. P. Mounfield, J. T. Hungerford and K. S. Walton, Ind. Eng. Chem. Res., 2015, 54, 12408-12414.

24 G. Férey, C. Mellot-Draznieks, C. Serre, F. Millange, J. Dutour, S. Surblé and I. Margiolaki, Science (80-. )., 2005, 309, 2040.

25 S. Øien, D. Wragg, H. Reinsch, S. Svelle, S. Bordiga, C. Lamberti and K. P. Lillerud, Cryst. Growth Des., 2014, 14, 5370-5372.

26 O. I. Lebedev, F. Millange, C. Serre, G. Van Tendeloo and G. Férey, Chem. Mater., 2005, 17, 6525-6527.

27 M. C. Bernini, D. Fairen-Jimenez, M. Pasinetti, A. J. Ramirez-Pastor and R. Q. Snurr, J. Mater. Chem. B, 2014, 2, 766.

28 R. C. Huxford, J. Della Rocca and W. Lin, Curr. Opin. Chem. Biol., 2010, 14, 262268.

29 S. Wuttke, S. Braig, T. Preiß, A. Zimpel, J. Sicklinger, C. Bellomo, J. O. Rädler, A. M. Vollmar and T. Bein, Chem. Commun., 2015, 51, 15752-15755.

30 T. Preiß, A. Zimpel, S. Wuttke and J. O. Rädler, Materials (Basel)., 2017, 10, 216.

31 D. Cunha, M. Ben Yahia, S. Hall, S. R. Miller, H. Chevreau, E. Elkaïm, G. Maurin, P. Horcajada and C. Serre, Chem. Mater., 2013, 25, 2767-2776.

32 S. Devautour-Vinot, C. Martineau, S. Diaby, M. Ben-Yahia, S. Miller, C. Serre, P. Horcajada, D. Cunha, F. Taulelle and G. Maurin, J. Phys. Chem. C, 2013, 117, 11694-11704.

33 R. Anand, F. Borghi, F. Manoli, I. Manet, V. Agostoni, P. Reschiglian, R. Gref and S. Monti, J. Phys. Chem. B, 2014, 118, 8532-8539.

34 P. Horcajada, F. Salles, S. Wuttke, T. Devic, D. Heurtaux, G. Maurin, A. Vimont, M. Daturi, O. David, E. Magnier, N. Stock, Y. Filinchuk, D. Popov, C. Riekel, G. Férey and C. Serre, J. Am. Chem. Soc., 2011, 133, 17839-17847.

35 N. Liedana, P. Lozano, A. Galve, C. Tellez and J. Coronas, J. Mater. Chem. B, 2014, 2, 1144-1151.

36 N. Liédana, E. Marín, C. Téllez and J. Coronas, Chem. Eng. J., 2013, 223, 714-721.

37 A. Chakraborty and C. Adhikari, Chemphyschem, 2016, 17, 1070-7.

38 H. Zheng, Y. Zhang, L. Liu, W. Wan, P. Guo, A. M. Nyström and X. Zou, J. Am. Chem. Soc., 2016, 138, 962-968.

39 N. Motakef-Kazemi, S. A. Shojaosadati and A. Morsali, Microporous Mesoporous Mater., 2014, 186, 73-79.

40 S. Wang, C. M. McGuirk, M. B. Ross, S. Wang, P. Chen, H. Xing, Y. Liu and C. A. Mirkin, J. Am. Chem. Soc., 2017, 139, 9827-9830.

41 R. Röder, T. Preiß, P. Hirschle, B. Steinborn, A. Zimpel, M. Höhn, J. O. Rädler, T. Bein, E. Wagner, S. Wuttke and U. Lächelt, J. Am. Chem. Soc., 2017, 139, 23592368.

42 J. Zhuang, C. H. Kuo, L. Y. Chou, D. Y. Liu, E. Weerapana and C. K. Tsung, ACS 
Nano, 2014, 8, 2812-2819.

43 A. Nehlig, J. L. Daval and G. Debry, Brain Res. Rev., 1992, 17, 139-170.

44 D. Hexsel, C. Orlandi and D. Zechmeister do Prado, Dermatol. Surg., 2005, 31, 866-872; discussion 872.

45 M. Nakagawa and K. Kawai, Contact Dermatitis, 1995, 32, 9-13.

46 M. Mohammadpour, M. Behjati, A. Sadeghi and A. Fassihi, Int. Wound J., 2013, 10, 260-264.

47 J. Nawarak, R. Huang-Liu, S.-H. Kao, H.-H. Liao, S. Sinchaikul, S.-T. Chen and S.-L. Cheng, J. Proteome Res., 2008, 7, 3737-46.

48 X. Liu, W. Xia, Q. Jiang, Y. Xu and P. Yu, J. Biosci. Bioeng., 2015, 120, 335-339.

49 L. Paseta, G. Potier, S. Sorribas and J. Coronas, ACS Sustain. Chem. Eng., 2016, 4, 3780-3785.

50 S. Tanaka, K. Kida, T. Nagaoka, T. Ota and Y. Miyake, Chem. Commun., 2013, 49, 7884-7886.

51 S. A. Moggach, T. D. Bennett and A. K. Cheetham, Angew. Chem., Int. Ed., 2009, 48, 7087-7089, Angew. Chem., 2009, 121, 38

52 Y. Hu, H. Kazemian, S. Rohani, Y. Huang and Y. Song, Chem. Commun., 2011, 47, 12694.

53 I. Khay, G. Chaplais, H. Nouali, C. Marichal and J. Patarin, RSC Adv., 2015, 5, 31514-31518.

54 K. W. Chapman, G. J. Halder and P. J. Chupas, J. Am. Chem. Soc., 2008, 130, 10524-10526.

55 I. Erucar and S. Keskin, Ind. Eng. Chem. Res., 2016, 55, 1929-1939.

56 M. J. Rosseinsky, A. Grigoropoulos, A. I. McKay, A. P. Katsoulidis, R. P. Davies, A. Haynes, L. Brammer, J. Xiao and A. S. Weller, Angew. Chem., Int. Ed.,2018, 77, 17; Angew. Chem., 2018, 130, 17.

57 C. Petit, Curr. Opin. Chem. Eng., 2018, 20, 132-142.

58 N. Liédana, A. Galve, C. Rubio, C. Téllez and J. Coronas, ACS Appl. Mater. Interfaces, 2012, 4, 5016-5021.

59 P. Serra-Crespo, A. Dikhtiarenko, E. Stavitski, J. Juan-Alcañiz, F. Kapteijn, F.-X. Coudert and J. Gascon, CrystEngComm, 2015, 17, 276-280.

60 S. M. J. Rogge, J. Wieme, L. Vanduyfhuys, S. Vandenbrande, G. Maurin, T. Verstraelen, M. Waroquier and V. Van Speybroeck, Chem. Mater., 2016, 28, 5721-5732.

61 S. M. J. Rogge, M. Waroquier and V. Van Speybroeck, Acc. Chem. Res., 2017, 51, 1, 138-148.

62 P. G. Yot, K. Yang, F. Ragon, V. Dmitriev, T. Devic, P. Horcajada, C. Serre and G. Maurin, Dalt. Trans., 2016, 45, 4283-4288.

63 C. M. Hansen, Hansen solubility parameters: a user's handbook, CRC Press, Boca Raton, 2nd edn., 2007.

64 B. H. Ye, X. Y. Li, I. D. Williams and X. M. Chen, Inorg. Chem., 2002, 41, 64266431.

65 M. M. Finnegan, T. G. Lutz, W. O. Nelson, A. Smith and C. Orvig, Inorg. Chem., 1987, 26, 2171-2176.

66 B. Seoane, S. Sorribas, Á. Mayoral, C. Téllez and J. Coronas, Microporous Mesoporous Mater., 2015, 203, 17-23.

67 S. Bourrelly, B. Moulin, A. Rivera, G. Maurin, S. Devautour-Vinot, C. Serre, T. 
Devic, P. Horcajada, A. Vimont, G. Clet, M. Daturi, J. C. Lavalley, S. Loera-Serna, R. Denoyel, P. L. Llewellyn and G. Férey, J. Am. Chem. Soc., 2010, 132, 94889498.

68 S. Devautour-Vinot, G. Maurin, F. Henn, C. Serre and G. Férey, Phys. Chem. Chem. Phys., 2010, 12, 12478-12485.

69 Z. Zhao, X. Li and Z. Li, Chem. Eng. J., 2011, 173, 150-157. 